Neurosurg Focus 22 (5):E5, 2007

\title{
Brain edema
}

\author{
Julian T. Hoff, M.D., Richard F. KeEP, Ph.D., ANd Guohua XI, M.D. \\ Department of Neurosurgery, University of Michigan Health System, Ann Arbor, Michigan
}

\section{$\mathrm{B}$} RAIN EDEMA HAS BEEN a therapeutic enigma for centuries. All practitioners who treat brain disorders routinely confront brain swelling resulting from water accumulation. However, successful management of edema and its bedfellow, raised intracranial pressure (ICP), is not an easy task.

The mechanisms underlying brain edema vary, depending on the type of brain insult. Classically, brain edema has been divided into cytotoxic (cellular), vasogenic, ${ }^{2}$ and interstitial (hydrocephalic) types. It should be noted, however, that many disease states are a combination of types. There is a great need for specific treatment to match the mechanism, but treatment modalities have not greatly changed in decades. More than a century ago, ventriculostomy for cerebrospinal fluid drainage and decompression craniotomy were used to control increased ICP resulting from mass effect and edema. Both treatments are still used clinically. Almost a century ago, serum osmolality manipulations were found to be effective in the laboratory. ${ }^{4}$ Later, hyperosmolar fluids were used clinically with good effect, and they remain a mainstay of edema treatment. Corticosteroids were used to treat tumor-associated edema for 50 years, ${ }^{3}$ but the efficacy of these agents is largely limited to edema associated with tumors or infection. Hyperventilation, assuming a $\mathrm{CO}_{2}$ reactive vascular bed is present, continues to be used to reduce ICP. Anesthetic agents to suppress brain blood flow and metabolism have also been used to control brain edema with modest success.

The difficulties in treating brain edema are exemplified in stroke. Stroke-induced edema involves changes in the vascular bed, blood-brain barrier integrity, and both extracellular and intracellular compartments. A single treatment has not been found for stroke edema and the ICP elevation that often follows.

In the current edition of Neurosurgical Focus, the authors aim to give readers a comprehensive overview of brain edema formation, current treatments, and potential future therapies. There are articles in which the authors deal with the basic mechanisms underlying cytotoxic and vasogenic edema (Marmarou and Liang et al.) and how an understanding of those mechanisms has led researchers to examine the roles of aquaporins (water channels: Bloch and Manley; Marmarou), matrix metalloproteinases (Rosenberg and Yang), and nonselective cation channels (Liang et al.) in edema formation. Such innovative approaches provide great hope for new therapies for brain edema.

In the next series of articles, the authors deal with edema by disease state. These articles cover edema caused by traumatic brain injury (Kawamata et al.) stroke (Thiex and Tsirka and Mocco et al.), and tumor (Stummer), including an interesting hypothesis exploring the relationship between edema and peritumoral cyst formation (Baggenstos et al.). Next, some of the significant advances in ICP monitoring and brain edema imaging are examined by Czosnyka et al. and Kuroiwa et al. Imaging, ICP monitoring, and comprehensive physiological monitoring provide a wealth of information on brain edema formation that may prove of great use in guiding therapy.

In the last section, the authors deal with medical and surgical treatments for brain edema. Current medical treatments are discussed, with a particular focus on the relative benefits of hypertonic saline compared with mannitol for osmotic therapy (Raslan and Bhardwaj). The potential for preconditioning with hyperbaric $\mathrm{O}_{2}$ to reduce intracerebral hemorrhage-induced brain edema is examined (Qin et al.). There has been much interest among basic scientists about the mechanisms by which preconditioning stimuli can reduce a variety of brain injuries. It may be possible to translate these findings to neurosurgery. ${ }^{1}$ Finally, the question of surgical treatment to alleviate the effects of brain edema is explored. There has been a longstanding debate over the benefits of surgical decompression. The current evidence on the utility of decompression for traumatic brain injury and stroke is reviewed (Hutchinson et al.).

\section{References}

1. Chan MT, Boet R, Ng SC, Poon WS, Gin T: Effect of ischemic preconditioning on brain tissue gases and $\mathrm{pH}$ during temporary cerebral artery occlusion. Acta Neurochir Suppl 95:93-96, 2005

2. Klatzo I: Presidential address. Neuropathological aspects of brain edema. J Neuropathol Exp Neurol 26:1-14, 1967

3. Kofman S, Garvin JS, Nagamini D, Taylor SG III: Treatment of cerebral metastases from breast carcinoma with prednisolone. J Am Med Assoc 163:1473-1476, 1957

4. Weed LH, McKibben PS: Experimental alteration of brain bulk. Am J Physiol 48:531-555, 1919

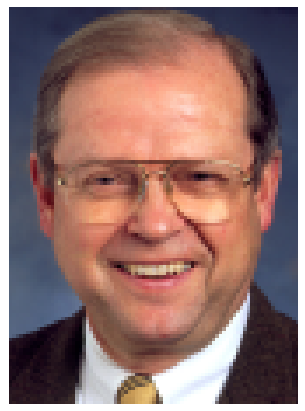

\section{In Memoriam}

After editing this issue of Neurosurgical Focus, Julian T. Hoff passed away on April 16, 2007. Dr. Hoff had an impact on the field of neurosurgery and brain edema research in many ways. He was a great clinician, teacher, and researcher, as well as an advocate for neurosurgery and medical research. Above all, he was a good man, and the world is much poorer for his passing.- R.F.K./G.X. 
Neurosurg Focus 21 (2):E?, 2006

A

Abbreviations used in this paper: 